\title{
Mária Huber \\ »Das ungarische Modell«: Von den Reformen zur Rezession
}

\section{Reformen}

»Bekanntlich ist Ungarn das einzige Land im RWG-Block, das eine marktorientierte Reform des zentralistischen Planungssystems sowjetischen Typs nicht nur im Jahre 1968 eingeführt hat, sondern das den Reformkurs bis zum heutigen Tage durchzuhalten vermochte $\ll$ (Kosta 1984, S. 228).

Ungarn schien lange die Ausnahme zu sein, Modell sogar für die anderen sózialistischen Länder: Privatinitiative und Profit behaupteten sich zunehmend gegen ideologische und institutionelle Bastionen der Planwirtschaft. Derartige Veränderungen in den Funktionsprinzipien des Wirtschaftsmechanismus erhob Wlodimierz Brus zum Kriterium von Reformen. Der polnische Ökonom im Exil grenzte die »Umstellung auf einen anderen Wirtschaftsmechanismus« von den vielfältigen Korrekturen ab, die seit 1965/66 - dem Beginn der sowjetischen Verwaltungsmaßnahmen zur Verbesserung wirtschaftlicher Effektivität - das Planungssystem in allen osteuropäischen Ländern mehr oder weniger modifizierten. Allerdings nur in Jugoslawien und - bei allen Differenzen und Inkonsequenzen - in Ungarn lösten geld- und finanzpolitische sowie betriebliche Entscheidungen staatliche Planbefehle ab (Brus 1985, S. 57). Als Brus im Jahre 1985 die im Westen weit verbreitete ordnungspolitische Auffassung von der Reformfähigkeit der sozialistischen Wirtschaft auf den Begriff brachte, befanden sich beide »Musterländer « auf der Schwelle von der latenten zur offenen Wirtschaftskrise (vgl. Völgyes 1987; Reuter 1984; 1985). Die »kritische Theorie« des »real existierenden Sozialismus « ging indessen durchweg von einer gedachten Realität aus und operierte nur mit prinzipiellen, nicht mit realpolitischen Alternativen. Stabilität und Krisenfestigkeit sahen die Neo-Reformisten nicht explizit als Zielsetzungen an. Denn dies waren seit Breschnjews Amtsantritt die Orientierungsmarken der Parteibürokratie, die sich radikalen Änderungen widersetzte. Der Weg ist daher - wie bei Bernstein - das Ziel: Nur sollen die Schritte diesmal in Richtung Dezentralisierung führen. Das aber ist eine erklärte Abwendung vom einstigen Glaubenssatz führender Sozialdemokraten und Lenins, der bis heute in der Politischen Ökonomie des Sozialismus kanonisiert ist.

Da die ungarische Dezentralisierung nicht auf einer umfassenden Reformkonzeption beruhte (Gumpel 1983, S. 197), blieb die Beurteilung derEntwicklung eine Sache der Weltanschauung. Einige Theoretiker (Osteuropäer zumeist) hofften auf einen neuen Sozialismus, andere hingegen warnten vor einer Überbewertung der durchgeführten Maßnahmen .

In Ungarn selbst hat die Reformdiskussion in den letzten zwei bis drei Jahren - angesichts der schon deutlich spürbaren Krise - an Schärfe und Spannung zugenommen. Die heutige Rück- 
schau auf die Wellen der Wirtschaftsreformen klammert die politische Perspektive nicht mehr (völlig) aus. Den Auftakt dazu gab ein Interview von RezsöNyers, des »Vaters der Wirtschaftsreformen«. Der ehemalige ZK-Sekretär (1962-1974) befaßte sich mit dem Widerspruch, daß die politische Führung neue Etappen und Elemente der Reform stets mit dem Hinweis auf die ideologische und institutionelle Kontinuität ankündigte. Sie blockierte damit sich selbst und schwächte die Wirkung der Reformmaßnahmen ab. Die aufgestauten Probleme verlangten jedoch, daß die Partei ihre führende Rolle für neue Reforminitiativen nützte ${ }^{2}$. Offiziellen Verlautbarungen zufolge suchte die Ungarische Sozialistische Arbeiterpartei (USAP) erst noch den Weg. Der Amtsantritt des neuen Generalsekretärs der KPdSU, Michail Gorbatschow, im März 1985 gab dazu keinen unmittelbaren Anstoß. In Ungarn dauerte es fast so lange wie im Westen, bis die Reformpläne der neuen sowjetischen Führung ernsthaft zur Kenntnis genommen wurden. Noch im Sommer 1986 beklagte Nyers die geringe Resonanz in der Fachund Tagespresse. Die reservierte Haltung kann nicht nur mit Skepsis gegenüber der sowjetischen Entwicklung erklärt werden. Gorbatschows Eintreten für »radikale Reformen« nahm den System-Konservativen das Alibi, wonach die Sowjetunion alle Reformansätze streng überwache und ihre Entfaltung begrenze.

Als die Grenzen für Reformen zum erstenmal erweitert wurden - das war 1953 nach Stalins Tod und nach den ersten Krisensignalen aus der DDR - reagierten maßgebliche Kräfte der Parteiführung unter Mátyás Rákosi ähnlich wie heute einige Bruderparteien: Wir müssen die Ideen der sowjetischen Genossen nicht kopieren und ihren Vorschlägen wie Sklaven folgen (Peto/Szakás 1985, S. 240 ff). Sie pochten auf ihre Eigenständigkeit, um - trotz Kritik und Korrekturen - bei den alten Methoden zu bleiben: Planen durch Willkür, Wirtschaften durch Zwang, Regieren durch Angst. Der Lohn der Angst war der Oktober-Aufstand 1956, in dem sich der lange aufgestaute Widerspruch zwischen Vision und Wirklichkeit des Sozialismus entlud. Die ökonomische und politische Krise stürzte das Land in bürgerkriegsähnliche Zustände. Um ihre Macht zu konsolidieren, mußte die - neugegründete - Partei von der (direkten) Ausübung einer Befehlsgewalt sofort Abstand nehmen. Zu ihren neuen Grundsätzen erklärte die veränderte Parteiführung bereits Anfang 1957 die Anerkennung unterschiedlicher Interessen in der Gesellschaft und die Demokratisierung der Leitungsprozesse. Die Aufgabe des Allmachtanspruchs zugunsten einer »Bündnispolitik « (szövetségi politika) bedeutete vor allem den Abbau des »übermäßigen Zentralismus«. Den Ausweg aus der »Parteikrise« - die laut Selbstkritik der USAP aus der »Geringschätzung oder sogar der Ignoranz der Verbündeten der Arbeiterklasse « folgte - sah die poststalinistische Regierung nicht in einer Reform des politischen Systems, sondern in der »Integration der Bevölkerung «. In zähen parteiinternen Kämpfen gelang es immerhin ansatzweise, dem stalinistischen Sozialismus-Modell eine Realpolitik entgegenzusetzen (Huber 1984, S. l ff.), die sich an den historischen Besonderheiten und konkreten Bedingungen Ungarns und nicht mehr an abstrakten »Gesetzmäßigkeiten der Entwicklung « orientierte. »Unser Postulat war «, sagte Politbüro-Mitglied György Aczél 1982 im Gespräch mit Francis Cohen, »daß der Sozialismus nicht nur eine Sache der Zukunft sei; auch diese Generation sollte die Früchte ihrer Arbeit genießen«. Der Begriff »Lebensstandardpolitik « (életszinvonal-politika) wurde zwar erst Mitte der sechziger Jahre geprägt, doch erste Maßnahmen zur Verbesserung der Versorgung traf die Kádár-Führung unmittelbar nach 
der Einleitung der Konsolidierungsphase: Das System der Zwangsabgaben wurden abgeschafft. Die Dezentralisierung begann. Die Regierung sah sich jedoch außerstande, die Reformkonzeption anzunehmen, die in ihrem Auftrag erarbeitet worden war. Ein Mitglied der damaligen Regierungskommission, József Bognár, erịnnerte knapp 30 Jahre später an das Übergewicht jener Kräfte, die bei der Renovierung der Machtverhältnisse lieber zum alten System zurückgekehrt wären (MV, H. 11/1986, S. 6).

Die theoretische Auswertung der Erfahrungen erbrachte folgende Reformbedingungen (vgl. Szamuely 1986; Bauer 1987):

1. Reformen werden aus Krisen geboren. Die politische Führung ist ohne massiven sozialen Druck nirgends geneigt, die Risiken einzugehen, die mit radikalen Änderungen der Prioritäten und der Machtverhältnisse verbunden sind. Dennoch führen selbst schwere Krisen nicht notwendigerweise zu Reformen. Notsituationen verleiten oft zu kurzfristigen Notlösungen und ergeben eine »Restriktions-Spirale« (L. Antal).

2. Eine Reform setzt neue Leitbilder und neue Handlungsmuster voraus. Dazu müssen entsprechende Vorschläge vorliegen. Ohne eine Emanzipation von der offiziellen Ideologie können Wirtschafts- und Sozialwissenschaften jedoch weder die Gründe der Disfunktionen untersuchen, noch brauchbare (und nicht schlichte Wunsch-) Vorstellungen für die Überwindung der Probleme entwickeln. Nur wenn führende Mitarbeiter des Staats- und Parteiapparates sowie der Forschungsinstitute zu einer (gewissen) Zusammenarbeit, also auch zu Kompromissen, bereit sind, kann ein Reformprogramm überhaupt zustande kommen. Dessen Verwirklichung setzt allerdings einen gesellschaftlichen Konsens voraus.

An dieser letzten Voraussetzung scheitern nach Ansicht von L. Szamuely und T. Bauer die gegenwärtigen Reformversuche in Polen. Ihrer Ansicht nach waren Mitte der sechziger Jahre auch in der Tschechoslowakei nicht alle gesellschaftlichen und politischen Bedingungen der Reform vorhanden. In Ungarn hingegen nahmen an der Reformvorbereitung zwischen 1963 und 1966 die Vertreter verschiedener sozialer Gruppen teil. Dies wurde durch die politische Entwicklung in den Jahren davor ermöglicht. Das Reformprojekt kam letztlich nach der Austragung (einiger) gesellschaftlicher Konflikte zustande. Deren Ergebnisse zeigten sich 1961/62 (MV, H. 11/1986, S.8):

- die Kollektivierung der Landwirtschaft, die zwar keineswegs problemlos verlief, aber die Grundlagen für die Anwendung moderner Produktionstechniken schuf;

- der Ausschluß von Rákosi und Gerö, zwei Schlüsselfiguren der stalinistischen Führung, aus der Partei, wodurch der dogmatische Block, dessen Basis im Mittelfeld der Bürokratie besonders stark war, entscheidend geschwächt wurde;

- ein neuerlicher Vorstoß reformorientierter Ökonomen, die nach einer defensiven Phase ihre Möglichkeiten erkannten, als die Unzufriedenheit mit der Erhöhung des Lebensstandards und mit der wirtschaftlichen Entwicklung allgemein spürbar wurde. Konsum war immerhin schon legitim.

1965/66 war schließlich die Bahn frei für die Vorbereitung und Diskussion einer Wirtschaftsreform. Im Zentrum der Auseinandersetzungen stand die Rolle von Plan und Markt im Wirtschaftssystem. Dezentralisierung und Monetarisierung wurden im gewissen Rahmen akzeptiert. Dennoch war es eine Sensation, als 1985 zum erstenmal ein Lehrbuch der Politischen 
Ökonomie erschien, das von der »neuen« Theorie ausging, wonach Sozialismus und Warenproduktion einander nicht ausschließen, sondern aufeinander abgestimmt werden können. Der Autor, Balázs Hámori, argumentierte, das Ziel von Wirtschaftsreformen müsse es sein, Marktverhältnisse zu schaffen. Dies sei in den vorausgegangenen zwanzig Jahren nicht geschehen (Hámori 1985, S. 330 ff.).

Der am 1. Januar 1968 in Kraft gesetzte »neue Wirtschaftsmechanismus« bestand im wesentlichen aus den Elementen, die schon in der Reformkonzeption von 1957 enthalten waren. Doch »keines der befreundeten sozialistischen Länder hat die Reformbestrebungen konsequent unterstützt«, berichtete unlängst der damalige Außenminister János Péter. Er gab sogar zu, daß nach der Prager Krise von 1968 ein Mitglied der polnischen Parteiführung äußerte, das nächste Land, in das einzumarschieren sei, werde Ungarn sein. Parteichef Gomulka habe sich allen Bestrebungen der sozialistischen Länder energisch widersetzt, diplomatische Beziehungen zur Bundesrepublik Deutschland aufzunehmen. Er fürchtete, die BRD werde dann die DDR und darauffolgend die NATO die osteuropäischen Staaten »anschließen«« (MV, H. 11/ 1986, S. 10).

Der "neue Wirtschaftsmechanismus mußte sich also in einer komplizierten innen- und außenpolitischen Situation behaupten. Ihr relativer Erfolg ist daher nicht zuletzt eben jener Versicherung der Parteiführung zu verdanken, die politische Machtstruktur werde stabil bleiben. Gänzlich unberührt von den Veränderungen blieb sie jedoch nicht. Die Reformpolitik ließ das zentralisierte Planungssystem - trotz mancher Rückschritte - immer weiter hinter sich (Bauer 1987, S. 533):

- Die Lenkungsinstrumente und -institutionen wurden reduziert, verpflichtende Plankennziffern gestrichen;

- die Verteilung von Ressourcen und Produktionsgütern ging von den zentralen Behörden an Handelsorganisationen über;

- Leistungsanreize und Gewinnorientierung blieben nicht länger an betriebs- und branchenspezifische Normative gebunden, sondern wurden von einem - prinzipiell zumindest einheitlichen - Preissystem gesteuert.

Der Parteibeschluß zur Reform des Wirtschaftsmechanismus (1966) erklärte das Staats- und Genossenschaftseigentum für gleichrangig und eröffnete damit Entwicklungsmöglichkeiten für die Landwirtschaft. Mit der Vorherrschaft des Genossenschaftseigentums in der Landwirtschaft war in den fünfziger Jahren das Argument verbunden, sie sei noch nicht richtig sozialistisch. Ideologie und Investitionspolitik ließen ihr daher mehr Maßregeln denn Mittel zuteil werden. Infolge der Modernisierung und der Marktorientierung stiegen Produktion und Einkommen im Agrarsektor Ende der sechziger Jahre an. Die Industriebetriebe verwandelte der neue Wirtschaftsmechanismus von Befehlsempfängern in eigenständig kalkulierende Anbieter von Waren. Dabei erwiesen sich vor allem die Großbetriebe als nicht rentabel (genug). Die Lohnentwicklung in der Industrie nahm sich - im Vergleich mit dem schnell wachsenden Wohlstand auf dem Lande, aber auch mit dem besseren und teuereren Warenangebot der Lebensmittelgeschäfte in den Städten - als recht bescheiden aus. Nach der traditioneilen Auffassung von Sozialismus waren jedoch Großbetriebe - die »Hochburgen« der Arbeiterklasse - per definitionem rentabel. Wenn es nun anders erschien, mußte nach dieser Logik der Fehler 
nicht bei den »führenden« Monopolbetrieben liegen, sondern in den Regeln des neuen Wirtschaftsmechanismus zu finden sein. Sie zu korrigieren bereitete schon deswegen keine besonderen Schwierigkeiten, weil die Investitionspolitik - die von den Dezentralisierungsmaßnahmen ausgenommen worden war-, die Rohstoff- und Schwerindustrie weiterhin vorrangig bediente. Auch die Lohn- und Preispolitik blieb früheren normativen Vorstellungen und sozialpolitischen Funktionen untergeordnet. Die Verteilung der Einkommen (Ressourcen und Revenue) erfolgte also weiterhin nicht nach ökonomischen Leistungs- und Rationalitätskriterien. Obwohl die Reform den Übergang zum intensiven Wirtschaftswachstum (höhere Produktivität, schnellere Innovation) hätte unterstützen sollen, pflasterten die verteilungs- und beschäftigungspolitischen Maßnahmen die alten Wege des extensiven Wachstums, die in der Industrialisierungsphase angelegt worden waren. Einen Fortschritt brachte der Reformkurs insofern, als eine gewisse Rationalisierung in den Verwaltungs- und Verhandlungsprozessen Voluntarismus und Willkür eindämmte. Als einen weiteren Schritt der Reform empfahl József Bognár 1970, eine neue Außenwirtschaftspolitik zu erarbeiten. Dahinter stand die Erkenntnis, daß die Wirtschaftstheorie des Sozialismus - von den Autarkiebestrebungen der Sowjetunion geprägt - nur einen Tauschhandel kannte, nicht aber die internationale Arbeitsteilung, also die aktive Teilnahme an den technologischen und organisatorischen Entwicklungstrends des Weltmarktes. Unter den führenden Ökonomen der damaligen Reformkommissionen obsiegte jedoch eine defensive Haltung, nach der nicht die Weiterentwicklung, sondern die $\gg$ Verteidigung der Reformen « das Gebot der Stunde sei. Denn inzwischen traten alle maßgeblichen Kräfte verbal für die Reformen ein, gegen deren Prinzipien die täglichen Entscheidungen, die sie trafen, so offenkundig verstießen. Die Gewerkschaften übten einen massiven Druck auf Öffentlichkeit und Parteiführung aus, wachsende Einkommensdifferenzen nicht zu tolerieren (MV, H. 11/1986, S. 11 ff.). Die Reformgegner suchten - und fanden - Rückendeckung in Moskau. Die KremlFührung regierte seit Beginn der siebziger Jahre unter dem Motto: »Keine Experimente.«Das innen- und wirtschaftspolitische Stabilitätsbedürfnis der sowjetischen Führung folgte auf Jahrzehnte großen Leids, verursacht durch Kollektivierung, Klassenkampf (Stalin) und Krieg (Hitler) sowie auf die Unsicherheit, die Chruschtschows sprunghafte Reformen für die Funktionärsschicht bedeuteten. Die achtzehn Breschnjew-Jahre - seit dem XXVII. Parteitag wegen »Stagnation « auch in der UdSSR kritisiert - gaben der sowjetischen Bevölkerung das Gefühl der Ruhe und existentieller Sicherheit. Diesen "positiven Effekt« (Szamuely 1986, S. 909) klammern Kritiker des »real existierenden Sozialismus « aus ihrer Betrachtung meist aus.

Die Destabilisierung, die Anfang der siebziger Jahre entstand, als in Ungarn die Reformkoalition angesichts erster Konflikte zerbrach, veranlaßte die Kreml-Führung im Februar 1972 von János Kádár die Überprüfung jener Maßnahmen zu verlangen, deren Konsequenzen - die Wiederherstellung kleinkapitalistischer Verhältnisse und die Vernachlässigung der sozialen Gerechtigkeit - die Konservativen in Budapest und Moskau gleichermaßen in Unruhe versetzten. Nach Kádárs Rückkehr aus Moskau bekamen jene Kräfte, die sich auf die Verteidigung der Arbeiterinteressen beriefen, starken Auftrieb. Auf der ZK-Sitzung im November setzten sie den (durch Kompromisse kaschierten) Abbruch des leistungs- und profitorientierten Wirtschaftskurses durch (vgl. Schmidt-Häuer 1986; Lendvai 1986, S. 74 ff.).

Die Verfechter der »Arbeiterpolitik « bestimmten die Reaktion auf den Ölschock und auf den 
Wachstumseinbruch in den kapitalistischen Ländern 1973/74. Unter der Führungsrolle der Partei flossen Dollar-Kredite in die ungarische Wirtschaft: zur Förderung des Wachstums, der Investitionen und des Konsums. Die Devise lautete, daß die sozialistische Wirtschaft durch die Veränderungen des Weltmarktes nicht in Mitliedenschaft gezogen werden dürfe. Wie in der Anfangsphase der Planwirtschaft, ging die Führung von Wunschvorstellungen aus, die mit der Realität unvereinbar waren. So suchte sie ihr Heil wieder in der Schaffung neuer Großbetriebe in Industrie und Landwirtschaft. Zwischen 1968 und 1980 verringerte sich die Zahl der Wirtschaftseinheiten von 9400 auf 3700 (HV vom 7.2.1987). Die künstlich entstandenen Monopole stärkten die Verflechtung von Unternehmen, Staats- und Kommunalorganen bis hinunter auf die lokale Ebene. Eine Trennung von Ökonomie und Politik hatte die Machtzentrale zu keiner Zeit angestrebt. Jetzt aber entstand ein unkontrollierter Lobbyismus, der die ohnehin fehlende Funktionsteilung zu seinen Gunsten nutzte. Das bedeutete, daß der produktive Sektor auf Kosten der Infrastruktur und der Sozialpolitik den Löwenanteil der Investitionsmittel und der staatlichen Steuerungskapazität beanspruchte (MV, H. 11/1986, S. 13 f.). »Das Zeitalter der Illusionen " (so Ungarns international angesehener Banker János Fekete) ging 1978 dramatisch zu Ende. Der Import-Überschuß erreichte 8-10\% des Volkseinkommens, die terms of trade (die Austauschrelationen zwischen Export- und Importpreisen) verschlechterten sich Jahr für Jahr, das hohe Importpreisniveau erhöhte den Inflationsdruck. 1979 versuchte die Regierung die Wirtschaft zu stabilisieren - mit einer »Vollbremsung «: Importe und Inlandsverbrauch wurden gedrosselt, Exporte hingegen - um jeden Preis! - gefördert. Gleichzeitig befürwortete der ZK-Beschluß vom Herbst 1979 die Weiterentwicklung der Wirtschaftsreform: Mehr Markt sollte für Prosperität sorgen. In den nächsten Jahren folgten - aus westlicher Sicht spektakuläre - Entscheidungen (Antal 1986, S. 127 ff.):

- Preisreformen (seit 1980), die im Binnenhandel Einflüsse des Weltmarktes simulierten und die Betriebe verpflichteten, die selbstkostenorientierte Preisbildung in eine marktorientierte umzuwandeln, während sich der Staat weiterhin vorbehielt, Preise zu kontrollieren bzw. zu subventionieren;

- Förderung der Privatinitiative (seit 1981) durch die Erleichterung von Pachtverträgen im Einzelhandel und im Gaststättengewerbe sowie durch die Zulassung von Arbeitskollektiven und Kleinunternehmen und am Rande des staatlichen Produktionssektors;

- Erweiterung der Entscheidungsautonomie der Unternehmen (seit 1985) im Bereich der Produktions- und Betriebsorganisation sowie des Kapitaltransfers - unter Mitwirkung eines gewählten Unternehmensrats (oder Managements), dem auch die Wahl des Direktors obliegt, während die Funktionen der Staatsorgane von der direkten Betriebsaufsicht auf eine Rechtsaufsicht reduziert wurden;

- Dezentralisierung des Bankensystems (seit 1985) durch die Trennung der Notenbank- und Geschäftsbankfunktionen und durch die Gründung neuer Geldinstitute wie z.B. Entwicklungsbanken als Gemeinschaftsunternehmen.

$\mathrm{Da} B$ diese Reformen nicht nur Vorteile versprechen, sondern auch harte Konsequenzen haben, wurde den Ungarn erst 1986 bewußt, als sich die Regierung endlich dazu durchrang, die Auflösung unrentabler Großbetriebe und die Unterstützung entlassener Arbeiter gesetzlich zu regeln. 


\section{Relikte und Raubbau}

Auf der Maikundgebung des Jahres 1987 sah sich Parteichef János Kádár gezwungen, die Hauptsorge des vergangenen Jahrzehntes deutlicher als früher anzusprechen: im Lande werde mehr verbraucht als produziert. Der schillernde Lebensstandard der Ungarn, den westliche Stammtisch- und Seminartheorien der Einführung von Marktelementen zuschreiben, beruht nur in Ausnahmefällen auf international wettbewerbsfähigen Leistungen. In Wahrheit ist der schöne Schein teuer erkauft: durch die Ausschöpfung aller erdenklichen Kreditquellen und der eigenen Reserven:

»Eine - im internationalen Vergleich - ansehnliche Wachstumsrate erreichte das Land in den fünfziger Jahren auf Kosten der Landwirtschaft, in den sechziger Jahren auf Kosten der Infrastruktur, in den siebziger Jahren durch Verschuldung. Drei Jahrzehnte lang begünstigte die weltweite Konjunktur die ungarische Wirtschaft. All dies erschien in der Selbstdarstellung allerdings als Beleg für die Überlegenheit des Systems. Die Konsequenzen dieser Disproportionen sowie der mißachteten Modernisierungsund Rentabilitätserfordernisse blieben lange Zeit verborgen, wofür der Preis jetzt zu zahlen sein wird.« (MN vom 9.5.1987)

Das Wirtschaftswachstum sank seit Beginn der achtziger Jahre noch weit stärker als durch die Stabilitätspolitik vorgesehen war. Die Produktion ging nicht nur in den unrentablen Betrieben und Branchen zurück. Bei kaum veränderter Wirtschaftsstrukturkam das Gros der Investitionsmittel weiterhin den Großbetrieben, der Rohstoff- und Schwerindustrie zugute. Für eine Modernisierung der verarbeitenden Industrie fehlte es an Kapital. Die Rentabilität der Investitionen blieb gering. Mit den Dollar-Krediten wurden die veralteten Produktionsstrukturen und der Konsum finanziert, nicht jedoch moderne Technologien. Allein bei den Konsumentenansprüchen erfolgte eine Anpassung an die moderne Weltwirtschaft (HV vom 9.5. 1987).

Die Notwendigkeit, Lücken in der Zahlungsbilanz zu stopfen, nahmen zentrale Behörden noch Mitte der achtziger Jahre zum Anlaß, in die Autonomie der Betriebe einzugreifen, ihnen - ohne Rücksicht auf die Rentabilität - Exportverpflichtungen aufzubürden. Zur Wahrung der Kreditwürdigkeit und des Versorgungsniveaus steuerte und subventionierte der Staat die Produktion in vielen Bereichen. Erfolgreiche Unternehmen mußten für die Aufrechterhaltung unrentabler Produktion bis zu zwei Drittel ihrer Gewinne in den Staatshaushalt abführen und konnten selbst nicht genug Investitionsmittel akkumulieren. Wirtschaftsplanung und -politik wurden mit dem notwendigen Strukturwandel nicht fertig. Sie schwankten zwischen Fortschreibung des Status quo und der Verordnung von Feuerwehreinsätzen zur Bewältigung der brennendsten Probleme.

In Ergänzung - teilweise im Gegensatz - zur ökonomischen Sichtweise, wonach ungelöste Zielkonflikte sowie die zunehmende »Inkonsistenz des Wirtschaftssystems« (E. Antal) die Rückschläge und Reibungsverluste erklären, meine ich, daß zwei Relikte des alten Sozialismus-Modells den Fortschritt blockieren: die Professionalisierungs- und die Integrationsmechanismen. Diese beiden Elemente der Produktionsverhältnisse behindern den Übergang vom extensiven zum intensiven Wirtschaftswachstum. Der Raubbau an den Produktivkräften kann - vorübergehend - staatliche Modernisierungsmaßnahmen ersetzen. Kern der Produktionsverhältnisse ist das staatliche Eigentum an Produktionsmitteln. Wer in welcher Form über sie verfügt, bestimmt die Partei. Bei der Besetzung der Kommandoposten in Wirtschaft und 
Verwaltung sind seit der Aufbauphase des Sozialismus politische Zuverlässigkeit bzw, proletarische Herkunft die entscheidenden Auswahlkriterien. Die Kaderpolitik der Partei ist zwar im Laufe der siebziger Jahre um die Kriterien Fachkompetenz und persönliche Führungsqualität erweitert worden. Die neuen Grundsätze brachten jedoch weder eine Verbesserung noch eine Demokratisierung der Rekrutierungsprozesse von Führungskadern. »Subjektivität « und »Spontaneität« bei den personalpolitischen Entscheidungen beklagten die offiziellen Stellungnahmen, ohne jedoch deren tiefere Ursachen zu nennen. Einzelne Beiträge in der theoretischen Monatszeitschrift der Partei »Társadalmi Szemle« befaßten sich zwar seit Beginn der achtziger Jahre mit dem Bildungsstand der Funktionäre, gaben aber nur vereinzelt Hinweise auf deren Ursachen. Denn die USAP war in der Kaderpolitik in ein Dilemma geraten. Die führende Rolle der Arbeiterklasse als Legitimationsprinzip konnte durch die Entwicklung der Mitgliederstruktur immer weniger gestützt werden: Der Anteil der Arbeiter nahm immer weiter ab. Die Beförderung von Arbeiterkadern in Führungspositionen schien daher allein geeignet, der Klassentheorie genüge zu tun. Ein Fern- und Abendstudium konnten die Kandidaten »nachholen «. Aber nur in Ausnahmefällen brachte der »zweite Bildungsweg « eine fachlich hinreichende - und gesellschaftlich akzeptierte - Qualifikation (vgl. Huber 1984, S. 63 ff.; 1985, S. 258 ff.).

Im März 1986 hat daher das Zentralkomitee der USAP neue Richtlinien beschlossen, wonach die Mehrzahl der Führungspositionen im staatlichen, wirtschaftlichen und kulturellen Leben durch Ausschreibungen und Wahlen besetzt werden sollen. »Der Führungsnachwuchs soll sich hauptsächlich aus Hochschul- und Universitätsabsolventen mit entsprechender Berufserfahrung rekrutieren« (DIE ZEIT vom 28. März 1986).

Den Beschluß begründete die Parteiführung mit der lange gereiften Erkenntnis, daß die weitere Entwicklung des Landes davon abhängig sei, ob es gelingen werde, die Qualifikation der Führungskräfte zu erhöhen. Denn trotz der großen Anzahl von Diplom-Ingenieuren und Ökonomen sind ein Drittel der oberen und zwei Drittel der unteren Führungspositionen in der Wirtschaft noch mit Nicht-Graduierten besetzt. Noch größer ist ihr Anteil in den Staatsorganen und in der Verwaltung. Besonders ins Auge fällt dabei, daß der Anteil von Graduierten gerade unter den jüngeren Führungskräften in einigen Bereichen sogar rückläufig ist. Die bisherige Praxis der Kaderpolitik schafft soziale Spannungen und wirkt demotivierend auf die Leistungsbereitschaft. Sie unterläuft damit alle sonstigen Bemühungen der Staats- und Parteispitze, Leistung und Effizienz als gesellschaftliche Normen durchzusetzen (Knopp/Radics 1984, S. 57; Veress 1985, S. 779). Vom Erfolg dieser Bemühungen hängt es entscheidend ab, ob Ungarn die zweite (und dritte) industrielle Revolution, die Phase des intensiven (und qualitativen) Wirtschaftswachstums erreicht. Andere Länder werden »nicht deshalb immer reicher, weil dort der Kapitalismus herrscht, sondern weil sie die Arbeit gut organisieren und ausführen « (Agoston 1985, S. 90). Im niedrigen Niveau der Arbeits- und Organisationskultur sehen ungarische Fachleute und Bürger einen - recht auffälligen - Grund für die unbefriedigenden Wirtschaftsergebnisse. Mit Disziplin und Präzision einerseits, mit Flexibilität und Kooperationsbereitschaft andererseits tun sich noch viele Beschäftigte schwer. Die Identifikation mit der Arbeit läßt viel zu wünschen übrig; dies gesteht auch die politische Führung ein. Mit Appellen und Anreizen versuchte sie gegenzusteuern. Allein, der Erfolg blieb gering. Wie tief das Problem sitzt, wird 
erst alimählich klar. Der Historiker Ferenc Glatz machte auf die bislang wenig beachteten Spätfolgen der Machtübernahme 1948/49 aufmerksam. Die neue Führungsschicht, die lauthals eine revolutionäre Politik verkündete, verstand wenig von den Lenkungsprozessen, die sie betrieb. In Wirtschaft und Verwaltung setzte die kommunistische Partei auf die Kraft von Parolen und auf die Mittel des Klassenkampfes. Mit ihren Mobilisierungskampagnen wertete sie nicht nur den Sachverstand ab, sondern die tägliche Arbeit schlechthin (Glatz 1986, S. 4).

Ins gesellschaftliche Bewußtsein prägte sich tief ein, daß die Leistung am Arbeitsplatz für das persönliche Aus- und Fortkommen eher nebensächlich sei, daß Sorgfalt und Initiative nicht belohnt werden. Den Modernisierungsprozeß erschwert und behindert bis zum heutigen Tage, daß Autorität durch Kompetenz weitgehend verloren ging. Im Sozialismusbild, das Ungarn übernahm, war die Wertschätzung intellektueller - und sogar jeglicher geistiger - Arbeit gegenüber der Rolle der Arbeiterklasse stark herabgesetzt (Kulesár 1985, S. 10, 20). Mißtrauen und Mißgunst gegenüber der gebildeten Elite verstärkten noch die vulgärmarxistische These, ihre Arbeit sei nicht produktiv. Diese Auffassung hat zu dem Resultat geführt, daß der Anteil der Hochschulabsolventen an der Bevölkerung wie auch der Anteil der white collar workers an den Beschäftigten im Vergleich zu den Industrieländern einen erheblichen Rückstand zeigen. Die zu eng spezialisierten akademischen Berufe genießen nur geringes Ansehen, sind schlecht bezahlt, stark feminisiert ${ }^{3}$.

Das Bild des Arbeitsmarktes gleicht bis ins Detail dem sowjetischen: Ärzte, Ingenieure, Ökonomen, Pädagogen und Biologen verdienen häufig weniger als Facharbeiter, in den ersten Berufsjahren sogar weniger als Hilfsarbeiter. Eine so geringe Bezahlung kann sich - angesichts der im Vergleich hohen Lebenshaltungskosten-ein Familienoberhaupt, ein Mann in der Regel, nicht leisten. Die Abwanderung der Akademiker in andere Berufe nimmt seit Anfang der siebziger Jahre ständig zu. Und nur 12\% der Jungen -- hingegen 24\% der Mädchen - besuchen ein Gymnasium, das zur allgemeinen Hochschulreife führt. Rund 80\% der Mittelschüler erwerben in den zwei- bis vierjährigen Fachschulen lediglich enge berufsspezifische Kenntnisse. Weder ihre Allgemeinbildung noch ihre berufliche Grundausbildung entsprechen den späteren Anforderungen. Wegen der engen Spezialisierung ist die geistige und räumliche Mobilität der gelernten Arbeitskräfte gering. Mobilität der Produktivkräfte wäre aber Voraussetzung für Strukturwandel. Das Bildungssystem ist also mitverantwortlich für die weitgehende Unfähigkeit und Unwilligkeit, mit technologischen und organisatorischen Innovationen Produktivität und Absatz zu steigern. Als eine weitere Bremse für den Strukturwandel erweist sich die alte Vorschrift, wonach die Betriebe verpflichtet sind, die Arbeitsplätze den amtlich entworfenen Berufsbildern anzupassen (Bessenyei 1987, S. 299 f.). Solche Relikte der direkten und detaillierten Wirtschaftslenkung, die dazu dienen, Angebot und Nachfrage am Arbeitsmarkt zu harmonisieren, bringen die Unternehmen von vornherein in eine Lage, in der es für sie unmöglich ist, selbständig zu wirtschaften. Die Leitung der Unternehmen muß den »oben « bestimmten Spielregeln - die obendrein oft unbestimmt und unbeständig sind - gerecht werden oder ihnen »irgendwie« ausweichen. Sich mit den unternehmerischen Aufgaben voll zu identifizieren, ist dabei nicht möglich (Mellár 1987, S. 340). Die Aufgaben selbst lassen eine rationale Geschäftspolitik nicht zu: Sie sind »uferlos« (Práger).

»Immer mehr ihrer Funktionen hat die Gesellschaft an die Unternehmen delegiert und diese mit den 
Folgekosten belastet. Anfang der achtziger Jahre waren die sozialistischen Betriebe der zentrale Ort der sozialen Fürsorge, der Politisierung, von Sport und Kultur, der menschlichen Beziehungen, der sozialen Existenz überhaupt« (MN vom 3.3.1987).

Der entscheidende Punkt ist, daß die Betriebe bei der Beschäftigung und Bezahlung der Belegschaft soziale und moralische Verantwortung zu tragen haben, weil eine autonome und aktive Sozialpolitik auf staatlicher Ebene erst in Ansätzen existiert. Die »Identitätsstörung « der Betriebe, bedingt durch die Unklarheit der Ziele und der Interessensverhältnisse, erweist sich als ein großes Hindernis für kompetentere Führungsmethoden. Die Management-Kultur ist auf einer niedrigen Stufe geblieben. Dynamische Persönlichkeiten haben es schwer (HV vom 7.2.1987). Obrigkeitsstaatliche Reglementierungen, die u.a. in den Personalabteilungen institutionalisiert sind, desorientieren und desinteressieren die Betriebswirtschaft auch »von innen« her. Eine Erhebung des Industrieministeriums dokumentierte vor kurzem, was bislang Tabu war: Besetzung und Arbeitsweise der Personalabteilungen folgen überholten Mustern aus der Frühphase des Sozialismus, als die Kaderfrage von den Wirtschaftsprozessen getrennt wurde. Lediglich in 59 Großbetrieben gibt es eine Direktion für Personal- und Sozialfragen, eine Organisationsform, die zurückgeht auf einen (nicht publizierten) Regierungserlaß aus dem Jahre 1976. An der alten Praxis änderte er im Grunde nichts (HV vom 11.7.1987). Mit den aus der »Gründerzeit « überlieferten Methoden der politischen Führung befaßt sich eine neuere Analyse von F. Kozma, Professor an der Parteihochschule: Die organisatorischen Lösungen »preßten die multifunktionalen und unendlich vielfältigen Integrationsbedürfnisse der Gesellschaft in ein Prokrustes-Bett: eindimensionale und hierarchische Regelungen versuchten die Kooperation zwischen Wirtschaft und Gesellschaft herzustellen.«

Allen Optimierungs- und Koordinationsbemühungen zum Trotz herrschen seiner Meinung nach vielfach anarchische Zustände, z.B. auf dem Wohnungsmarkt. Zu den generellen Folgen einer auf ökonomischen Illusionen und ideologischen Fixierungen beruhenden Integrationsstrategie, welche die Autonomie und die Selbstorganisation der sozialen Kräfte erdrosselte, gehöre zum einen die partielle Verarmung der Gesellschaft, zum anderen die geduldete (oder kriminalisierte) Mißachtung der Verwaltungsvorschriften (Kozma 1987, S. 32 ff.) »Das Programm kann nicht einfach die Erhöhung der (betrieblichen) Autonomie sein. Die Wege der modernen Integration müssen ausgebaut werden«, - forderte der Wirtschaftsjurist 'T. Sárközy schon 1983. Doch obwohl »die Politik die Koordinationsfunktionen des Marktes umfangreich übernahm« (Bihari) - nachdem die Wirtschaftsreformen der privaten und kollektiven Wirtschaftstätigkeit einen breiteren Raum eröffneten -, hat sie deren Segmentierung und Perspektivlosigkeit auf keiner der Steuerungsebenen zu überwinden vermocht:

- Die Grundsätze der Besteuerung sind - wie in den anderen sozialistischen Ländern - uneinheitlich. Das Einkommen aus dem Hauptberuf und im sozialistischen Sektor ist begünstigt. Das Haupteinkommen aus privater Arbeit ist mit hoher Steuer belastet. (Havasi 1987, S. 15 f.).

- Die Wirtschaftslenkung hat Bedingungen geschaffen, die von den Betrieben verlangen, in drei Markt-Kategorien zu denken: Inland, Dollarmarkt, Rubelmarkt. Die Betriebe können darüber hinaus nicht auf den Markt gehen, der für sie am günstigsten ist. Denn zentrale Interventionen modifizieren die Marktbedingungen $\gg$ im Interesse der sog. gesamtgesellschaftlichen Ziele«, und zwar nicht einheitlich, sondern unternehmensspezifisch (HV vom 23.5.1987). 
- Ein Pseudo-Zentralismus herrscht auch auf der obersten Ebene, wo mehrere Lenkungszentren funktionieren, isoliert voneinander (Antal 1985).

Die Heterogenität der zentralen Steuerung wird der Komplexität der sozialen und ökonomischen Prozesse nicht gerecht. Sie belastet überdies Bürger und Betriebe, macht sie doch ihre Dispositionsfreiheiten unsicher - und dadurch perspektivlos. Zugleich ist das bestehende System administrativer Politik funktional notwendig, da materielle Politikbereiche wie Verkehr und Kommunikation eklatante Mängel zeigen.

»Nach dem Krieg ist deren Entwicklung und Erweiterung vernachlässigt worden: Das statische und hierarchische Lenkungssystem bedurfte nicht einmal aus dem produktiven Sektor permanenter Rückmeldungen und hielt den Ausbau eines Informations- und Kommunikationsnetzes im sozialen Sektor bzw. einen umfangreichen und unkontrollierbaren Informationsfluß keineswegs für nötig, eher noch für gefährlich «".

In der gesamten »Reformzeit« war der Staat mehr damit beschäftigt, den Markt zu simulieren, denn öffentliche Güter und wohlfahrtsstaatliche Leistungen bereitzustellen, die zur Entfaltung wie zur Korrektur der Marktprozesse notwendig sind. Der Rückstand der Infrastruktur zu den entwickelten Industrieländern wird jetzt auf 40-50 Jahre geschätzt. In den vergangenen zwei Jahrzehnten wurde ihre Leistungsfähigkeit nicht im Gleichschritt mit dem produktiven Sektor ausgebaut. Auch dies ist eine Folge der Produktionsverhältnisse. Die Träger der sozialen Infrastruktur, die regionalen und kommunalen Körperschaften haben ihre Position im Entscheidungsprozeß - trotz nominell wachsender Autonomie - nicht ausbauen können. Sie sind auf Fürsprache und Finanzen seitens des Parteiapparats und der zentralen Behörden angewiesen. Für »nicht produktive« Investitionen hatte die auf extensive Produktion und auf kurzfristige Erfolge programmierte Wachstumspolitik jedoch meist nur »den Rest« erübrigen können (Bélley 1984; Bogár 1983). Ungenügende Transport- und Kommunikationsverbindungen verursachen heute enorme Reibungs- und Rentabilitätsverluste. Allein der Schaden, den das viel zu kleine und häufig gestörte Telefonnetz verursacht, wird auf 11-12 Milliarden Forint jährlich geschätzt. Dem Entwicklungsstand entsprechend müßten für hundert Einwohner 22-23 Anschlüsse zur Verfügung stehen-anstelle der durchschnittlich 14 (in Budapest 35, in den Randgebieten 5). Zweitausend Dörfer - fast ein Viertel der Bevölkerung - haben nur zu Bürozeiten eine telefonische Verbindung zur Außenwelt (HV vom 1.11.1986; MH vom 20.10.1986; MN vom 11.10.1985). Die Ungarischen Eisenbahnen können wegen technischer Mängel Tausende von Waggons nur bei herabgesetzter Geschwindigkeit in Betrieb halten. Nur ein Drittel der Eisenbahnstrecke ist bislang elektrifiziert. Desolate Zustände und Investitionslücken führen zur Wiederbelebung administrativer Zuteilungsmaßnahmen. So mußte z.B. Anfang 1985 ein eigens dafür gebildetes Komitee Dringlichkeit und Reihenfolge der Transportaufträge festlegen (HV vom 19.7.1986 und vom 25.10.1986).

Segmentierung fördert Selbstversorgung (und umgekehrt). Der Anteil des Eigenverbrauchs beträgt in den ungarischen Unternehmen durchschnittlich $60 \%$ (in modernen Betrieben dagegen nur 40\%) der Produktion. Nicht zuletzt wegen der noch »weithin üblichen Unpünktlichkeit und Unzuverlässigkeit der Lieferungen bemühen sich alle Unternehmen, möglichst viele Vorprodukte und Werkzeuge in eigener Regie herzustellen « (HV vom 14.2.1987). Das System örtlicher Wirtschaftsbeziehungen ist zerrüttet. Die Unternehmen wissen nicht, was Koopera- 
tion ist (P, H. 8-9/1986, S. 109 und H. 11/1986, S. 59). Bei diesem Stand der Arbeitsteilung können Professionalität und Rentabilität nur mühsam bzw. nur durch weitere Unternehmenskonzentration - die wiederum kontraproduktiv ist - gesteigert werden. Mit den privaten Haushalten ist es ganz ähnlich bestellt. Dank der Hof- und Hilfswirtschaften - als ein Element der Marktorientierung im Westen hochgelobt - versorgen sich Arbeiter- und Angestelltenfamilien in hohem Maße selbst mit Lebensmitteln. Auch diese Produktion erfolgt zu Lasten von Rentabilität und Professionalität. Der private Zeit- und Energieaufwand ist wesentlich höher als in den staatlichen Agrarbetrieben. Und sofern die private Feld- und Gartenarbeit nicht Freizeitbeschäftigung sondern materielle Notwendigkeit ist, geht sie auf Kosten der Leistungsbereitschaft und der Konzentrationsfähigkeit am Arbeitsplatz ${ }^{5}$. Nach Schätzungen ungarischer Ökonomen und Soziologen lebt nur ein Viertel der Haushalte ausschließlich vom Lohn und Gehalt. Sie verdienen entweder überdurchschnittlich gut oder haben absolut keine Chance, etwas dazu zu verdienen. Von den rund 5 Millionen Arbeitnehmern gehen etwa 4 Millionen einer zweiten Beschäftigung nach. Viele treibt es nach Statussymbolen, andere müssen ein Haus bauen, weil sie weder am Wohnungsmarkt noch im - auf ein Minimum reduzierten - sozialen Wohnungsbau zum Zuge kommen. Eine weitere Gruppe der Nebenverdiener rekrutiert sich aus Berufsanfängern und Hilfsarbeitern, deren Mindestlohn unter dem oder um das Existenzminimum (3 000 Forint) liegt. (Ein Tribüneplatz beim Formel 1-Rennen kostet zwischen 1800 und $4000 \mathrm{Ft}$.) Durchschnittsverdiener (rund 6000 Forint) werden durch die sinkenden Reallöhne (seit 1978 sind die Lebenshaltungskosten im Jahresdurchschnitt um über $8 \%$ gestiegen) gezwungen, eine Nebeneinnahmequelle aufzutun. Nach den statistischen Daten verbrachten die Ungarn 1984 genau 9,1 Milliarden Stunden an ihrem Arbeitsplatz. In der legalen »zweiten Wirtschaft « - so schätzten die Statistiker -- wurde 4,5 Milliarden Stunden gearbeitet. Vor 15-20 Jahren erreichte die Gesamtarbeitszeit 9,9 bzw. 3,3 Milliarden Stunden. Das war insgesamt etwa eine halbe Milliarde Stunden weniger als heute (P, H. 12/1985). Während also die produktiven Kapazitäten der Industrie- und Agrarbetriebe - von wenigen Ausnahmen abgesehen - sich nicht verbessern, sondern wegen der Überforderung verschlechtern, müssen die Arbeitskräfte immer mehr leisten, um den Lebensstandard halten zu können. Die tägliche Arbeitszeit hat sich auf 12-14 Stunden verlängert. Am 1. Januar 1984 standen rund $74 \%$ der Frauen im arbeitsfähigen Alter (16-55) in einem Beschäftigungsverhältnis, gut drei Prozentpunkte mehr als vier Jahre zuvor. Teilzeitbeschäftigung ist nur für 1 Prozent der Frauen möglich.

Gesundheit und Familien sind dabei schweren Belastungen ausgesetzt. Der Zerfall menschlicher Beziehungen, Schwierigkeiten bei der Anpassung an die Gesellschaft und andere sozialpsychologische Probleme lassen die Krankheitsraten ansteigen: Jeder ungarische Staatsbürger sucht im Jahr fünfzehnmal einen Arzt auf (MV vom 7.2.1987 und vom 16.3.1987). Die Lebenserwartung der Männer ist auf weniger als 66 Jahre gesunken. Die Sterblichkeit der 40-49jährigen Männer hat sich zwischen 1964 und 1984 - aufgrund vieler und (außer dem hohen Alkoholkonsum) nicht eindeutig klärbaren Gründen - verdoppelt (Klinger/Monigl 1985, S. 27). Die Zunahme von Sozialisationsstörungen bei Kindern, Symptome wie Jugendkriminalität und Drogenkonsum beunruhigen Eltern und Pädagogen ${ }^{6}$. Im Jahre 1984 ging der Mikrozensus auch erstmals der Frage nach, wie berufstätige Frauen ihre Kinder versorgen. Nach diesen 
Daten verbringt knapp ein Drittel der 11-14jährigen die schulfreien Stunden des Tages ohne Aufsicht. Jeden Sommer befassen sich Zeitungen mit den Problemen, die sich daraus ergeben, $\mathrm{da}$ in den langen Schulferien viele Kinder die meiste Zeit allein bleiben. Ein Linguistik-Professor macht die unterbrochene Kommunikation zwischen den Generationen für die zunehmend zu beklagenden groben Umgangsformen, für das Sinken der Kommunikationskultur verantwortlich: Die Kinder - in Tagesstätten untergebracht oder auf der Straße - lernen die Sprache voneinander, nicht von den Erwachsenen. Wer dann aus der Welt der Gleichaltrigen in das öffentliche Leben eintritt, kann seine Gedanken und Gefühle nur unzureichend ausdrükken. An diesen sozialen Prozessen zeigt sich die Wirkung von Reformen und Rückschlägen, die den Wirtschaftsmechanismus veränderten ebenso wie die Wirkung von »non-decisions« in der Wirtschafts- und Gesellschaftspolitik. Dezentralisierung ohne Integration, Marktorientierung ohne Infrastruktur, Anspruchshaltung ohne Risikobereitschaft gefährden den Reproduktionsprozeß: Die ungarische Gesellschaft lebt von der Substanz.

\section{Rezession und Reaktionen}

Die Krisenzeichen mehren sich: Seit Anfang der achtziger Jahre verschlechtert sich die Wirtschaftslage, und das Vertrauen in die staatliche Wirtschaftspolitik und Steuerung schwindet. Mit diesem Urteil begründen Reform-Experten wie L. Antal und seine Mitarbeiter ihre Vorschläge für die Politik (Antal u.a. 1987, S. 642 ff.). Zunächst die Fakten:

Stagnierendes Wirtschaftswachstum und hohe Inflationsraten ergeben eine Stagflation. Sie unterscheidet sich nur dadurch von der Krisenform der kapitalistischen Länder, daß sie nicht mit hoher Arbeitslosigkeit, sondern mit sinkender Effektivität der Ressourcen - Arbeit und Kapital - einhergeht. Es wird also mit Verlusten produziert. Volumen und Rentabilität der Investitionen sinken, und ebenso die Wirtschaftsmoral. Verträge und Versprechungen - letztere spielen immer noch eine große Rolle in den horizontalen wie in den vertikalen Wirtschaftsbeziehungen - halten viele Betriebe und Privatproduzenten nicht ein. Kurzfristiges Denken beherrscht die Entscheidungen. Bereicherung - oder: wie im Falle betrieblicher Lohnkonflikte die Befriedigung ungerechtfertigter Ansprüche - haben Vorrang vor Investitionen und Innovationen?. Diese Probleme führen ungarische Fachleute und Funktionäre auf den Umstand zurück, daß Markteinflüsse immer noch zu wenig zur Geltung kommen:

- Geldinstitute, die dem - für einen Strukturwandel unerläßlichen - Kapitaltransfer dienen, haben zwar Mitte 1987 auch eine Börse für Wertpapierhandel gegründet. Doch das Problem der Eigentümerrechte ist auch nach der Trennung der Staatsverwaltung von der Wirtschaft nicht gelöst (Sárközy 1986). Einige Großunternehmen sind zwar zerteilt worden; Eigeninteresse ist entstanden, aber langfristiges, unternehmerisches Denken läßt sich ohne freie Verfügung der Kollektive über die Produktionsmittel nicht »einführen«.

- Kleinunternehmer können im Innovationsprozeß keine Vorreiterrolle spielen. Erstens sind viele auf die »Selbständigkeit « nicht gut vorbereitet. An kaufmännischen Kenntnissen fehlt es ebenso wie an einer breiten fachlichen Grundausbildung. Zweitens können sie im bestehenden Steuersystem Investitionen in modeme Technologien nicht als Kosten abschreiben. Mit 
Einzelverordnungen wurde die Steuerlast der »Privaten« in den letzten Jahren erheblich erhöht. Die ungeklärte und unsichere Eigentumslage hemmt die Investitionsbereitschaft vieler Kleinunternehmer. Nur wenige der »Arbeitsgemeinschaften « entwickeln eine Marktstrategie, viele ziehen es vor, einen großen Auftraggeber zu beliefern und nehmen die Abhängigkeit hin. Dieses Ergebnis einer Erhebung erklären die Forscher nicht zuletzt mit dem Fehlen einer Interessenvertretung (KS H 7-8/1987 S. 957 ff., MN vom 28.1.1986; HV vom 26.7.1986; N vom 20.8.1987).

Mit den Dezentralisierungs- und Reformmaßnahmen haben Staats- und Parteiführung ihr Ziel nicht erreicht: Wachstumskapazität und Wettbewerbsfähigkeit der Wirtschaft haben sich nur punktuell verbessert, der Inlandsverbrauch liegt über dem Leistungsniveau. »Infolgedessen, « so konstatierte der Vize-Präsident des Planamtes Miklós Pullai: »ob dem Land bis 1990 Wachstum, Stagnation oder Rezession ins Haus steht, ist schon keine Frage der Planung mehr, sondern deren Konsequenz (HV vom 1.8.1986). Zwei Konsequenzen sind bereits eingetreten: - Der Dollarsaldo der Zahlungsbilanz hat einen neuen Rekordstand erreicht; die terms of trade verschlechtern sich weiter; die Verschuldung ist so hoch, daß Ende des Jahrzehnts Zinsen und Tilgungen die Exporterlöse bis auf einen kleinen Rest aufbrauchen werden. Nicht nur wissenschaftlicher Pessismismus, sondern auch die praktisch-politische Einschätzung der Lage sieht die Gefahr, daß Ungarn auf dem Weltmarkt weiter an die Peripherie abgedrängt und auf die Stufe eines Entwicklungslandes zurückfallen wird (ebenda und Kozma 1987).

- Der Staatshaushalt schloß 1986 mit einem Rekord-Defizit von 47 Milliarden Forint (HV vom 1.8.1987). Das war mehr als doppelt so hoch wie »geplant«, und es war nicht das erste Mal so. 1986 kam die Differenz weitgehend den Krisen-Sektoren als Subvention zugute. So konnten zwar Arbeitsplätze gesichert werden, nicht gewährleistet ist aber die soziale Sicherheit der Rentner. Der Realwert der Renten sinkt infolge der Inflation und der zu geringen staatlichen »Kompensation«.

Auf die Alarmzeichen der Destabilisierung reagierten Staats- und Parteiführung im Frühjahr 1987 mit einem Aktionsprogramm, das unter anderem vorsieht:

- die Beschleunigung des technischen Fortschritts durch erleichterte Kreditvergabe und Steuerrückerstattung für erfolgreiche Unternehmen;

- die Senkungen der Kaufkraft durch Abbau der Preissubventionen, worauf im Juli Preiserhöhungen folgten;

- die baldige Einführung der Mehrwertsteuer und einer allgemeinen Einkommenssteuer, mit dem Ziel die Einnahmestruktur des Staatshaushalts zu verändern: Bislang kamen $80 \%$ der Einnahmen aus der Wirtschaft; die Mehrwertsteuer wird das ändern - und weitere Preiserhöhungen verursachen.

Die Sanierung der Wirtschaft erfordert harte Opfer von der Bevölkerung und harte Entscheidungen von den Politikern. Die bisherige Reformpolitik hatte "pragmatischen« Charakter. Ohne eine Konzeption, zwischen Routine und Ratlosigkeit einer "Zwangslaufbahn« folgend, ergaben sich »halbe Lösungen « aus einem Entscheidungsmuster, das Konflikte vermeiden und verschleiern wollte. Die »Konfliktvermeidungs-Strategie«, die zum Symbol der »Einheit« wurde, trug Elemente des alten Sozialismus-Bildes, das eine harmonische und homogene soziale Entwicklung projizierte (Hankiss 1986). Nach dem heutigen Diskussionsstand zeich- 
net sich $a b$, daß eine Reformkonzeption zwei Grundprobleme angehen muß: ein historisches und ein systemspezifisches.

Erstens: Um die ökonomische und politische Rückständigkeit, die das Land seit Anfang des 19. Jahrhunderts in der »Neurose« befangenhält, den Westen aufzuholen, ist eine konsequente Modernisierungsstrategie erforderlich. Zu ihren wichtigsten Aufgaben gehören der Ausbau der Infrastruktur, die Anerkennung von Kompetenz und die Hebung des Bildungsstandes (Kopátsy 1986; Hankiss 1986; Kulesár 1985 u.a.).

Zweitens: Um in die intensive Phase ökonomischen Fortschritts eintreten zu können, müssen Politik und Wirtschaft getrennt und Institutionen der Interessensvertretung entwickelt werden. Der Abbau des Zentralismus und ein neues System der sozialen Arbeitsteilung sowie der Integrationsmechanismen setzen ein neues Sozialismusbild voraus (Szamuely 1986). Zwischen diesen erreichenbaren Zielkomplexen gibt es Verbindungen. In der »Stellungnahme des ZK der USAP zum Programm der wirtschaftlichen und gesellschaftlichen Entfaltung « vom 2. Juli 1987 setzt die Revision der Wirtschafts- und Parteipolitik bei den gemeinsamen Elementen der »doppelten Entwicklungsstrategie« an: Fachleute, Intellektuelle und Führungskräfte erhalten mehr Verantwortung und mehr politische und materielle Anerkennung. Die »führende Rolle der Arbeiterklasse « wird nicht mehr erwähnt. Dies erleichtert wiederum die Differenzierung zwischen Vollbeschäftigung und effektiver Beschäftigung. Das Recht auf Arbeit heißt nicht mehr »Recht auf den Arbeitsplatz«, sondern Mobilität.

Mit diesen - vorerst nur angekündigten - Reformschritten steht die ungarische Führung nicht mehr allein ${ }^{8}$. Der Generalsekretär der KPdSU, Michail Gorbatschow, erklärte von Anfang an: »Ich brauche die Intelligenz«. In seiner Rede vor dem ZK-Plenum Ende Juni 1987 gebrauchte er auch den Begriff: effektive Beschäftigung. Bereits zuvor zeigte sich aber, daß sein Perestrojka-Programm nicht nur die Privilegien derFunktionärsschicht, sondem auch die Privilegien der Arbeiter antastet und auf entsprechenden Widerstand stößt (vgl. Schmidt-Häuer/Huber 1987). Ungarn geben seinen Vorschlägen um so eher eine Chance, ohne Reformen »revolutionären Ausmaßes« (Gorbatschow) auszukommen, da die theoretische Vorbereitung für die Umgestaltung des ökonomischen und politischen Systems weiter fortgeschritten ist. 


\title{
Anmerkungen
}

1 Worin diese bestanden, ist gut dokumentiert und erklärt in: Grothusen, K.-D. (Hg.) (1987): Südeuropa Handbuch V: Ungarn, Göttingen und in Schönfeld, R. (Hg.) (1985): Reform und Wandel in Südosteuropa, München.

2 Mozgó Világ, 1984, H. 2 S. 5-17; deutsch in: Südosteuropa, 1984, H. 7-8, S. 447-459.

3 Huszár, T.: »Nem középiskolás fokon ...«, Müveltség, közmüvelódés, értelmiség. Budapest 1981, S. $140 \mathrm{ff}$.

Das Durchschnittseinkommen junger Akademiker erreicht erst im 29-30. Lebensjahr das Lohnniveau von Facharbeitern. Bis zu diesem Alter haben sie nur $60 \%$ von dem verdient, was ihre nichtstudierten Altersgenossen erhielten und holen den Rückstand erst um das 45. Lebensjahr ein. Vg1.: Diplomás fiatalok a társadalomban, Budapest KSH, 1984, S. 38 f. »Wir müssen uns zu der Erkenntnis durchringen, daß wir in den vergangenen 10 - 15 Jahren der Schaffung jener politischen und ökonomischen Bedingungen nicht genug Aufmerksamkeit schenkten, die dem moralischen und vor allem dem materiellen Wertverlust technischer und geistiger Kreativität und Kompetenz hätten entgegenwirken können« ZK-Sekretär Pál Lénárd in: Népszabadság vom 31.12.1986.

4 Akademi-Mitglied T. Vámos, zitiert in Heti Világgazdaság vom 10.12.1983.

5 2,5 Milliarden Stunden haben Kleinproduzenten gebraucht, um - mit manueller Arbeit und primär für den eigenen Bedarf - ein Drittel des landwirtschaftlichen Bruttosozialproduktionswertes zu schaffen; zw̌̀ei Drittel erwirtschafteten die Großbetriebe in/mit 1,5 Milliarden Arbeitsstunden. Der Stundenertrag der Kleinproduzenten lag damit Anfang der 80er Jahre weit unter dem des professionellen Agrarsektors. Dennoch stieg die Zahl der Arbeiter und Angestellten, die ihre Zeit und Geld in die bäuerliche Wirtschaft investierten und deren Interessen eher der Gartenarbeit denn dem Beruf galt. Vgl.: Timár, J.: Idó és munkaidó, in: Közgazdasági Szemle 1985, H. 11, S. 1310.

6 Die Statistiken der letzten Jahre weisen wesentlich mehr jugendliche Straftäter auf, vgl. Magyar Nemzet vom 13.6.1986 und Népszabadság vom 17.7.1986. Die Zahl der Drogenabhängigen wird auf 30-35 000 geschätzt, vgl. Heti Világgazdaság vom 15.8.1987, S. 62.

7 Magyar Nemzet vom 28.3., 9.7.1987, Népszabadság vom 20.8.1987, Heti Világgazdaság vom 2.8. 1986, vom 7.2., 25.7.1987. Etwa 200 Unternehmen verbrauchten auch die Abschreibungen für Lohnerhöhungen, kritisiert Magyar Nemzet vom 21.6.1986.

8 Die Prognose von S. Bialer (1987, S. 128 f.), die aus der Überschätzung des »ungarischen Modells« resultierte, hat sich schnell überholt, vgl. Bialer, S.: The Soviet Paradox, London 1987, S. $128 \mathrm{f}$.

\author{
Abkürzungsverzeichnis \\ HV Heti Világgazdaság \\ KS Közgazdasági Szemle \\ MH Magyar Hirlap \\ MN Magyar Nemzet \\ MV Mozgó Világ \\ N Nepszabadság \\ P Pártélet
}




\section{Literatur}

Agoston, M. (1985): Önismeretiink és a technikei müveltség, in: Társadalmi Szemle, 1985, H.5

Antal, L. (1985): Gazdaságirányitási és pénzügyi rendszerünk a reform utján, Budapest

Antal, E. (1986): Betrachtungen zur ungarischen Wirtschaftsreform 1980-1985, in: Osteuropa 1986, H. 2, S. 127-137

Bauer, T. (1987): A gazdasági mechanizmus továbbfejlesztése vagy reformja?, in: Kozgazdasági Szemle, H. 5, S. 538 ff.

Bélley, L. (1984): Gazdaságirányitás és infrastrukturafejlesztés, Budapest

Bessenyei, I. (1987): Gazdaság, szakmarendszer, iskolarendszer, in: Kösgazdasági Szemle, 1987, H. 3 ,

Brus, W. (1985): Socialism - Feasible and Viable?, in: New Left Review, 1985, September-October

Bogár, L. (1983): A fejlódés ára, Budapest

Glatz, F. (1986): Az emberek viszonya munkájukhoz, politikájukhoz, nemzetükhöz, in: Valóság 1986, H.4

Grothusen, K.-D. (Hg.) (1987): Ungarn, Göttingen

Gumpel, W. (1983): Sozialistische Wirtschaftssyysteme, München

Hamori, B. (1985): A szocialista gazdaság elmélete, Budapest

Havasi, F. (1987): Az uj nyugdijrendszer és az adóreform elvei, in: Társadalmi Szemle, 1987, H. 6

Huber, M. (1984): Die Ungarische Sozialistische Arbeiterpartei. Binnenstruktur und Funktionsprobleme. Berichte des Bundesinstituts für ostwissenschaftliche und internationale Studien, Köln

Huber, M. (1985): Der XIII. Parteitag der Ungarischen Sozialistischen Arbeiterpartei, in: Südosteuropa, 1985, H. 5, S. $258 \mathrm{ff}$.

Huszár, T. (1981): »Nem Középiskolás fokon ...«, Müveltség közmüvelódés, értelmiség, Budapest

Klinger, A./Monig1, I. (1985): Gondolatok népesedéspolikánk távlati koncepciójáró1, in Társadalmi Szemle, 1985, H. 11

Knopp, A./Radics, K. (1984): A fiatal értelmiség helyzetéról, in: Társadalmi Szemle, 1984, H. 7-8

Kosta, J. (1984): Wirtschaftssysteme des realen Sozialismus. Probleme und Perspektiven, Köln

Kozma, F. (1987): A gazdaságfeijlesztés társadalmi vetületei, in: Társadalmi Szemle, 1987, H. 6

Kulcsár, K. (1985): A magyar szociológia történetszemlétete, in: Valóság, 1985, H. 5

Lendvai, P. (1986): Das eigenwillige Ungarn, Zürich

Mellár, T. (1987): »A hiány« és a szocializmus gazdaságtana, in: Közgazdasági Szemle, 1987, H. 3

Petó, I./Szakács, S. (1985):A hazai gazdaság négy évtizedének története, Budapest

Reuter, J. (1984): Zur Wirtschaftslage Jugoslawiens, in: Südosteuropa, H. 2, S. 113-126

Reuter, J. (1985): Sinkender Lebensstandard in Jugoslawien, in: Südosteuropa, 1985, H. 7-8

Schmidt-Häuer, Ch. (1974): in: DIE ZEIT, Nr. 22 vom 24.5.

Schönfeld, R. (Hg.) (1985): Reform und Wandel in Südosteuropa, München

Szamuely, L. (1986): Az europai KGST-országok gazdasági reformjainak kérdéséhez a 80-as évek derekán, in: Közgazdasági

Vgl.: Timár, J. (1985): Idó és munkaidó, in: Közgazdasági Szemle, 1985, H. 11

Veress, P. (1985): Helyünk a nemzetközi munkamegosztásban, in: Közgazdasági Szemle, 1985, H. 7-8

Völgyes, I. (1987): Ungarn: Steht eine Krise bevor?, in: Osteuropa, 1987, H. 5 


\section{Thomas Voß \\ Polen: Reformrhetorik gegen Konservatismus und Stagnation}

Die aktuelle polnische Reformpolitik wurde nicht von der jüngsten Entwicklung in der Sowjetunion veranlaßt, sondern hat ihre eigene Geschichte. Unter dem Schlagwort eines »polnischen Weges zum Sozialismus w wurde schon Mitte der 50er Jahre von der Parteiführung eine grundlegende Systemreform propagiert. Dies geschah in Reaktion auf eine umfassende gesellschaftliche Oppositionsbewegung, die sich aus tiefster Unzufriedenheit mit dem sozialistischen System stalinistischer Prägung entwickelt hatte. Niedriger Lebensstandard und eklatante Versorgungsmängel, härteste Arbeitsbedingungen und höchste Leistungsanforderungen, allgemeine Rechtsunsicherheit, Inkompetenz und Willkür der parteilich-staatlichen Machtapparate, ein tiefempfundenes Gefühl individueller wie gesellschaftlicher Machtlosigkeit gegenüber all diesen Erscheinungen, das Fehlen jeglicher authentischer Kommunikationsstrukturen und Möglichkeiten der Artikulation eigener Interessen und Ansichten waren Probleme, mit denen sich weite Teile der Bevölkerung nicht abfinden wollten. Hinzu kam, daß den Polen das sozialistische System sowjetischen Typs gegen ihren Mehrheitswillen mit Gewalt aufgezwungen worden war, und zwar durch die russische Großmacht, die sie traditionell als einen der gefährlichsten Feinde der polnischen Nation ansahen. Um das Herrschaftssystem gegenüber der gesellschaftlichen Bewegung aufrechtzuerhalten, bekannten sich die Machthaber verbal zu einer Reformpolitik, die den Bedürfnissen der Gesellschaft weit entgegenkam und grundlegende Freiheitsrechte, politische Mitwirkungsmöglichkeiten, Rechtsstaatlichkeit und materiellen Wohlstand in Aussicht stellte. Die Realisierung blieb jedoch weit hinter den Versprechungen zurück. Im wesentlichen blieb das System unverändert, so daß die Bevölkerung allmählich den Glauben an seine Reformierbarkeit verlor, ohne allerdings eine konkrete Alternative zu sehen, die ihrer allgemeinen Unzufriedenheit eine Perspektive geboten hätte.

Im Normalfall ist im realsozialistischen System die Gesellschaft atomisiert und aller authentischen Strukturen beraubt. Sie ist durchsetzt von hierarchischen Strukturen, die letztendlich von der obersten Spitze der Partei kontrolliert und gesteuert werden und keine andere Funktion haben als die, die Machtpositionen der Nomenklatur zu sichern. Von dieser Norm weicht Polen ab. Hier stellt die katholische Kirche eine gesellschaftliche Institution dar, die selbst unter den Bedingungen offenen Terrors in der Stalinzeit nicht gleichgeschaltet oder vernichtet werden konnte. Als authentische Organisation konnte sie seit Mitte der 50er Jahre ihren gesellschaftlichen Einfluß ständig vergrößern. Sie wurde geradezu zum Rückgrat der Gesellschaft, zum Kristallisationskeim gesellschaftlicher Selbstorganisation und andererseits zur Gegenmacht gegen die parteilich-staatliche Allgewalt der die Macht monopolisierenden Nomenklatura. Sie trug damit wesentlich zu der Entwicklung bei, die die gesellschaftliche Oppositionsbewegung allmählich zu immer stärkerem Selbstbewußtsein und immer deutlicheren Vorstellungen über ihre Ziele führte. Als im August 1980 die polnische Arbeiterklasse quasi als gesellschaftliche 